\title{
Combining Charge Couple Devices and Rate Sensors for the Feedforward Control System of a Charge Coupled Device Tracking Loop
}

\author{
Tao Tang ${ }^{1,2, *}$, Jing Tian ${ }^{1,2,3}$, Daijun Zhong ${ }^{1,2}$ and Chengyu Fu ${ }^{1,2}$ \\ 1 Institute of Optics and Electronics, Chinese Academy of Science, Chengdu 610209, China; \\ abb1978@163.com (J.T.); zqfirefly@126.com (D.Z.); cyfu@ioe.ac.cn (C.F.) \\ 2 Key Laboratory of Optical Engineering, Chinese Academy of Sciences, Chengdu 610209, China \\ 3 University of Chinese Academy of Sciences, Beijing 100039, China \\ * Correspondence: prettang@gmail.com; Tel.: +86-28-8510-0191
}

Academic Editor: Vittorio M. N. Passaro

Received: 23 April 2016; Accepted: 21 June 2016; Published: 25 June 2016

\begin{abstract}
A rate feed forward control-based sensor fusion is proposed to improve the closed-loop performance for a charge couple device (CCD) tracking loop. The target trajectory is recovered by combining line of sight (LOS) errors from the CCD and the angular rate from a fiber-optic gyroscope (FOG). A Kalman filter based on the Singer acceleration model utilizes the reconstructive target trajectory to estimate the target velocity. Different from classical feed forward control, additive feedback loops are inevitably added to the original control loops due to the fact some closed-loop information is used. The transfer function of the Kalman filter in the frequency domain is built for analyzing the closed loop stability. The bandwidth of the Kalman filter is the major factor affecting the control stability and close-loop performance. Both simulations and experiments are provided to demonstrate the benefits of the proposed algorithm.
\end{abstract}

Keywords: sensor fusion; charge couple device; feedforward control; time delay; light of sight error, FOG

\section{Introduction}

A direct feedback loop is usually utilized to control LOS in a CCD-based tracking system [1-3]. High control bandwidth facilitates better closed loop performance. However, limited sampling frequency and time delay are the major reasons to restrict the bandwidth. Time delays, namely including exposure time of the CCD, image process time and transmit time, cannot be cut to zero, resulting in ineffectiveness of the high bandwidth. The Smith predictor is introduced into the closed-loop system to compensate for time delays [4]. Experiments verify rate feed forward control to effectively improve the tracking performance, especially for a maneuvering target tracking $[2,5]$. How to obtain LOS rate is a major task to implement a feed forward control, because a tracker such as CCD cannot provide target trajectories or even target velocities but only the target error. LOS rate estimation usually synthesizes LOS error, encoder and rangefinder in a stationary platform [6]. An inertial measurement unit is required if estimating the LOS rate on an inertial stabilization platform [7]. Only employing LOS error and gimbal position was developed to generate LOS rate for compensation of LOS error [8]. As far as LOS rate estimators are concerned, there are many researchers who have developed new methods although they could not be used for feed forward control [9-11]. The Kalman filter is used to implement these estimation algorithms, especially for tracking a maneuvering target. Some papers focus on optimizing the Kalman filter to adapt to maneuvering targets [12-15]. However, the closed-loop stability is not taken into account when the LOS rate is not available. This paper 
proposes combining the CCD and FOG to recover the target trajectory as observed values of a Kalman filter which can produce the LOS rate to implement feed forward control. Additive feedback loops are inevitably added to the original control loops because some closed-loop information is utilized. The closed-loop stability and robustness are investigated on the condition of gain margin and phase margin of the open-loop transfer function with feed forward control. To analyze the closed-loop stability, the transfer function of the Kalman filter in the frequency domain is built.

Section 2 presents a detailed introduction to feed forward control based on sensor fusion, mainly describing the implementation of feed forward control; Section 3 focuses on parameter design, to be specific in terms of tracking controller and the Kalman filter; Section 4 discusses and analyzes system stability and sensitivity function; Section 5 sets up simulations and experiments to testify the theorems above; concluding remarks are presented in Section 6.

\section{Feedforward Control Based on Sensor Fusion}

The configuration of the CCD-based tracking system is a two-axes gimbals design illustrated in Figure 1. The sensors include FOG and a CCD. The FOG gyroscope mounted on the gimbal is usually used as the feedback component of the velocity closed loop. The controller is used to implement the control algorithm. The driver actuates the motors to achieve the tracking control. The light source is used to simulate the target of the CCD.

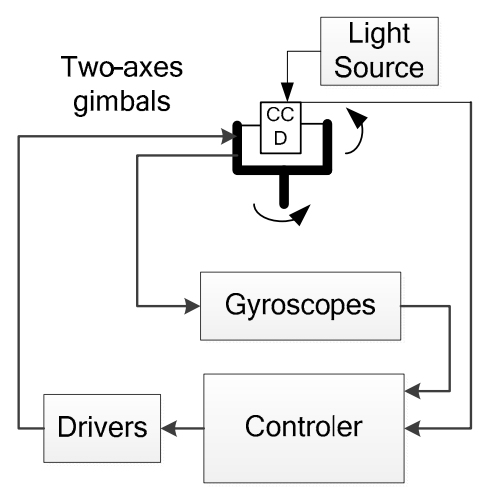

Figure 1. Configuration of the tracking control system.

The control mode of Figure 1, which includes two closed loops (position loop and velocity loop) and feed forward loop is shown in Figure 2. $Q(s)$ is the feedforward controller. $G(s)$ is the control plant. $C(s), C_{v}(s)$ are the position controller and the velocity controller. The time delay $e^{-T_{0} s}$ characterizes the $\mathrm{CCD}$ in the control system although it may be rough. $\mathrm{R}$ represents the target trajectory. $\mathrm{E}$ is the line of sight error. $\mathrm{O}$ is the output of the fiber-optic gyroscope (FOG), which provides the gimbal rate. A FOG providing the angular velocity of the gimbal usually has high bandwidth, resulting in a little effect towards to the closed-loop bandwidth. Therefore, the characteristic of the gyro can be considered to constant one in this control system.

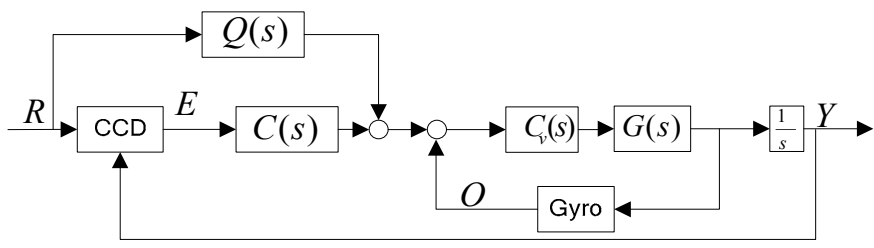

Figure 2. Classical feed forward control. 
The perfect feed forward control requires $Q(s)=s P^{-1}(s)$. The time delay cannot be compensated, so the perfect controller is expected to implement $Q(s) \approx s P^{-1}(s) . P(s)$ called the velocity closed-loop transfer function and is defined as follows:

$$
P(s)=\frac{C_{v}(s) G(s)}{1+C_{v}(s) G(s)}
$$

As a matter of fact, this term $\frac{C_{v}(s) G(s)}{1+C_{v}(s) G(s)}$ is very close to constant at low frequencies because the velocity closed loop has much higher bandwidth than that of the position closed loop. Thus, $P(s)$ $\approx 1$ is true to some extent. However, it is still impractical to implement $Q(s)=s P^{-1}(s)$, because the term $P(s)$ includes not only non-nominal part, but also high-frequency characteristics. In this case, the feedforward controller can be described as:

$$
Q(s)=\frac{s}{1+T_{f} s}
$$

The phase lag term $1 /\left(1+T_{f} s\right)$ indicates the main feature of a filter.

In a CCD-based control system, the CCD only provides LOS error while R is not available, so an equivalent control structure of Figure 2 is depicted in Figure 3.

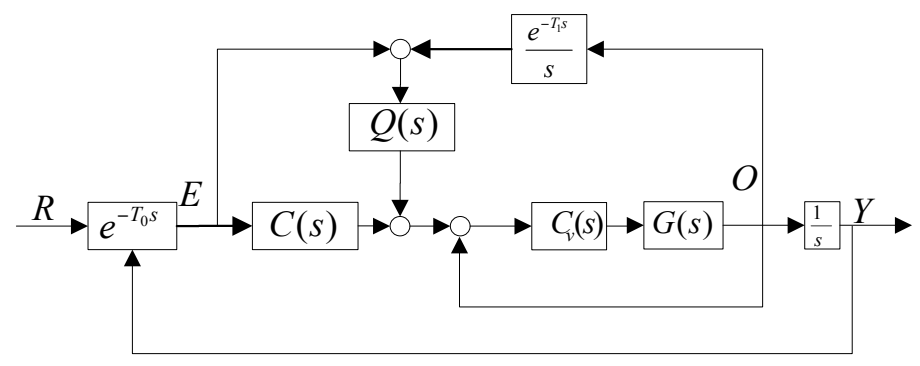

Figure 3. An equivalent feed forward control.

This control structure in Figure 3 is practical. This equivalent feed forward control combines the the CCD and rate sensor to recover the target trajectory produce the LOS rate to implement feed forward control. From Figure 3, we have:

$$
R=E+\frac{e^{-T_{1} s}}{S} O
$$

The time delay $e^{-T 1 s}$ is used to match the feature of delay about CCD. It is impossible to set $T_{1}=T_{0}$, because the time delay of CCD is uncertain although the sample frequency of FOG can reach several thousand Hertz or more, while the CCD usually has a frequency of dozens of $\mathrm{Hz}$. Differentiating the synthesizing signal $E+\frac{e^{-T_{1} s}}{s} O$ produces the line of sight rate due to bad noise, resulting in ineffectiveness. Therefore, a Kalman filter is used to estimate the line of sight rate in this paper. Before this, how the realized $\mathrm{R}$ as the observed value of the Kalman filter is the first step.

The bandwidth of the FOG is above $500 \mathrm{~Hz}$. The characteristics of the FOG noise are depicted in Figure 4 (Figure 4a represents the original signal, while Figure 4b describes the integration signal). The peak value of the FOG noise is equal to $0.025^{\circ} / \mathrm{s}$, and the RMS value is about $0.0037^{\circ} / \mathrm{s}$.

Besides some "spikes", the amplitude value of amplitude-frequency curve is smooth in Figure 5 (Figure 5a represents the FFT of the original signal, while Figure 5b describes the FFT of the integration signal). Especially, the integration of FOG signal is smoother due to the integral impact. 


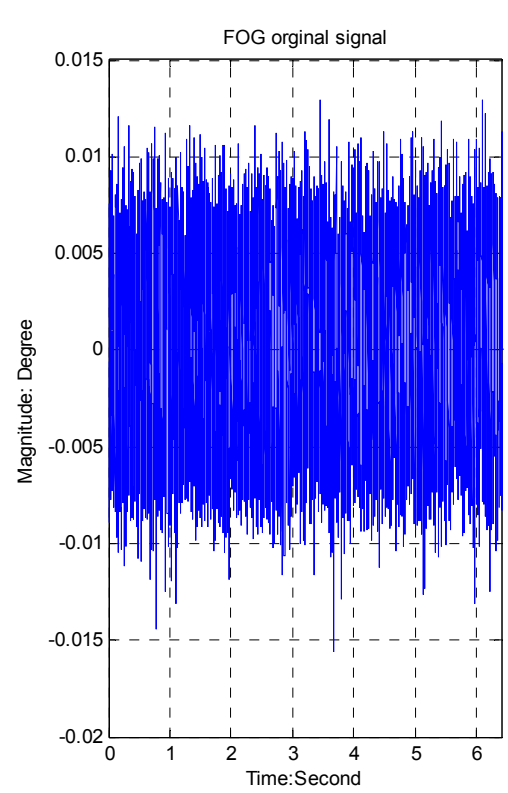

(a)

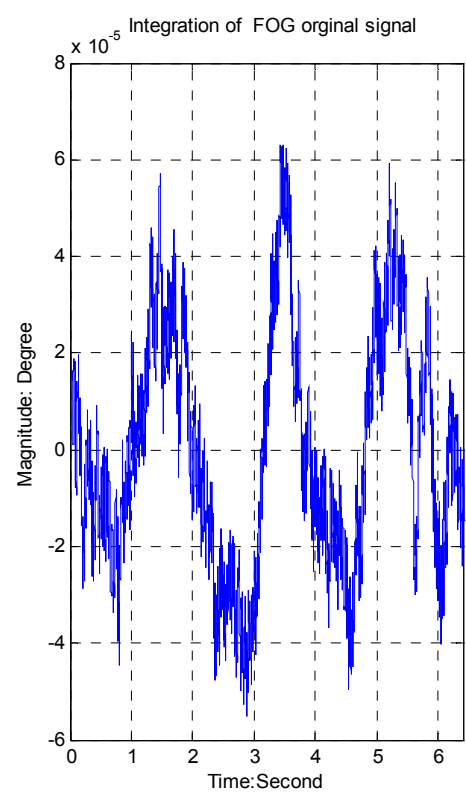

(b)

Figure 4. FOG Noise Characteristics FOG Noise Characteristics (a) The FOG original signal; (b) The integration signal of the FOG.

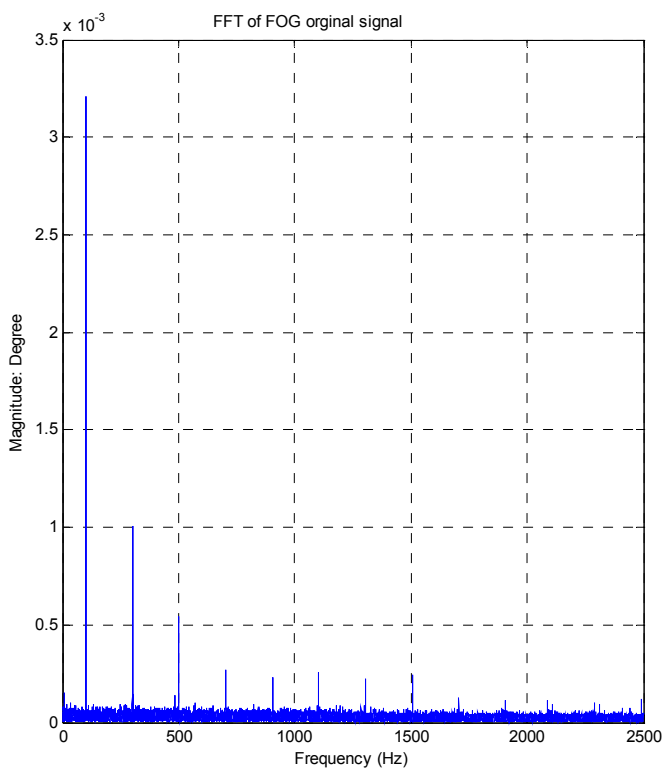

(a)

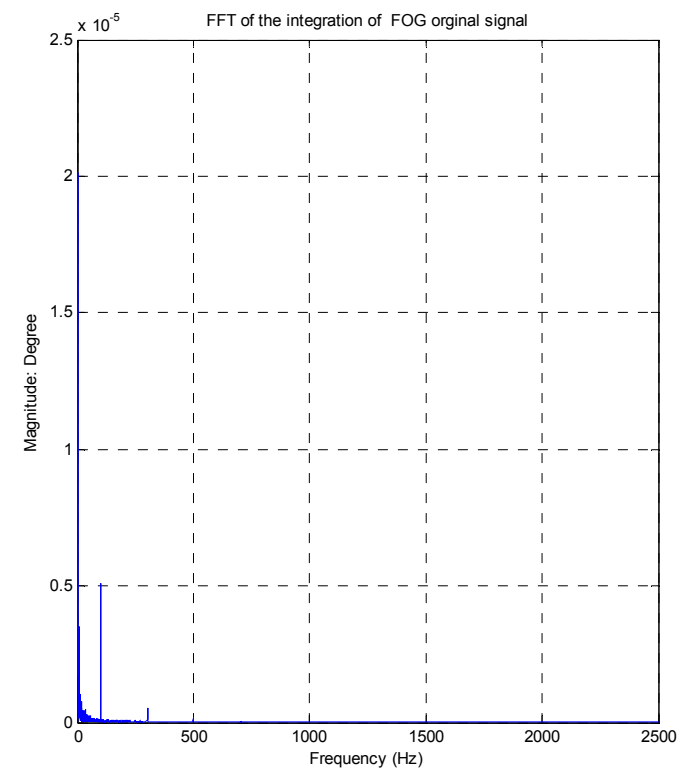

(b)

Figure 5. FOG FFT analysis (a) FFT of the original signal; (b) FFT of the integration signal.

The CCD characteristic in this system is shown in Table 1.

Table 1. CCD parameters.

\begin{tabular}{cc}
\hline Frame Frequency & $50 \mathrm{~Hz}$ \\
Pixels & $640 \times 512$ \\
Pixel Size & $5.5 \mu \mathrm{m}$ \\
Focus Length & $30-450 \mathrm{~mm}$ \\
\hline
\end{tabular}


The maximum focus length is $450 \mathrm{~mm}$, so the instantaneous field of vision is about $12.2 \mathrm{u}$ rad.

The integration of FOG signal is depicted as follows:

$$
\text { Int_FOG }(i+1)=O * \Delta t+I_{n} \_F O G(i), \quad i=0,1,2,3 \cdots
$$

where $\Delta t$ is the sampling time of CCD. Therefore, the precision of the integration of FOG is about $0.02 \times 0.025=0.0005^{\circ}$, smaller than $12.2 \mathrm{u}$ rad. Combining Equations (3) and (4), we have:

$$
R=E+\text { Int_FOG }(i+1)
$$

\section{Parameters Design}

The Kalman filter is employed to implement the filter $Q(s)$, because it is an optimal LMS filter to suppress noise [12]. The standard Kalman equations are depicted as follows:

$$
\left\{\begin{array}{c}
x_{k+1}=A x_{k}+B u+w_{k} \\
y_{k+1}=C x_{k}+v_{k}
\end{array}\right.
$$

The observed value of Kalman filter is $E+\frac{e^{-T_{1} s}}{s} O$, while the output value is $x_{k+1}(2)$, called target velocity. A mode called Singer acceleration model is used to calculate the differential signal [13]. The Kalman filter's parameters are as follows:

$$
A=\left[\begin{array}{ccc}
1 & T & 0.5 T^{2} \\
0 & 1 & T \\
0 & 0 & 1
\end{array}\right], B=\left[\begin{array}{lll}
\frac{1}{6} T^{3} & \frac{1}{2} T^{2} & T
\end{array}\right]^{T}, C=\left[\begin{array}{lll}
1 & 0 & 0
\end{array}\right]
$$

The process variance $Q_{k}$ is defined as to $Q_{k}=B \times B^{T} \times \sigma_{w}$, where $\sigma_{w}$ is variance of the observed value. The measurement variance $R_{k}$ is defined as to $\sigma_{v}$, which is the variance of sensor noise. The solution of the Kalman filter is below:

$$
\hat{x}_{k+1}=\left(A-K_{k+1} C A\right) \hat{x}_{k}+K_{k+1} y_{k}
$$

The gain $K_{k+1}$ of the Kalman filter can be obtained from the Ricatti equation if the matrices A, $\mathrm{B}$ and $\mathrm{C}$ are time-invariant and known. The reconstructive characteristic of the Kalman filter in the frequency domain can be described by Equation (8):

$$
\phi=\left(Z I-A+K_{k+1} C A\right)^{-1} K_{k+1}
$$

The sampling frequency of the CCD is $50 \mathrm{~Hz}$. The time delay $T_{0}$ is $0.06 \mathrm{~s}$. The open-loop transfer function without feed forward control is shown below:

$$
\frac{e^{-T_{0} s}}{s} C(s) P(s) \approx \frac{K_{p}\left(K_{I} s+1\right)}{s} \frac{e^{-T_{0} s}}{s}
$$

For the feedback system to be robust, a gain margin larger than $6 \mathrm{~dB}$ and a phase margin larger than 35 degrees is usually specified [16], so the PI controller parameters can be obtained as $K p=0.07096 / T_{0}^{2}, K_{I}=7.1541 T_{0}$.

The open-loop transfer function in Figure 2 is given by Equation (10):

$$
S_{\text {open }}=\frac{Q(s)+C(s)}{1-Q(s) P(s) \frac{e^{-T_{1} s}}{s}} P(s) \frac{e^{-T_{0} s}}{s}
$$


The requirements of the closed-loop system with feedforward control need to meet a phase margin larger than $35^{\circ}$ of the open-loop transfer function, so we have:

$$
\arg \left[\left.S_{\text {open }}(s)\right|_{s=j w_{c}^{\prime}}\right]+180^{\circ} \geqslant 35^{\circ}
$$

From the above equation, we can obtain $T_{f} \geqslant 0.195$. The bandwidth of the filter is limited to about $1 /(0.195 \times 2 \pi)=0.814 \mathrm{~Hz}$.

\section{Performance Analysis}

The sampling time is $T=0.02$, the measurement variance is $\sigma_{v}=0.1$ and the process variance is $\sigma_{w}=100$. Therefore, a Kalman filter with a bandwidth of $0.99 \mathrm{~Hz}$ is preferable for the control system, since the phase margin $(\mathrm{Pm})$ and the gain margin $(\mathrm{Gm})$ of the open-loop transfer Equation $(4)$ are $40.3^{\circ}$ at the frequency of $1.33 \mathrm{~Hz}$ and $8.71 \mathrm{~dB}$ at the frequency of $3.69 \mathrm{~Hz}$, respectively. The sensitivity function illustrated in Figure 3 is:

$$
S_{S F}=\frac{1-Q(s) P(s) \frac{e^{-T_{1} s}}{s}}{1+C(s) P(s) \frac{e^{-T_{0} s}}{s}+\frac{1}{s} Q(s) P(s)\left(e^{-T_{0} s}-e^{-T_{1} s}\right)}
$$

The sensitivity function without feedforward control is shown below:

$$
S_{S F}^{\prime}=\frac{e^{-T_{0} s}}{1+C(s) P(s) e^{-T_{0} s}}
$$

Based on the aforementioned considerations and design, the responses of the sensitivity Equations (12) and (13) are shown in Figure 6. A large attenuation, about one tenth less than that with only feedback loop control, is achieved in the low-frequency region with the feedforward controller in Figure 6. However, the attenuation in the middle-frequency range is magnified from $0.3 \mathrm{~Hz}$ to about $1.4 \mathrm{~Hz}$, a little bigger than that achieved with the only feedback control. This is due to the amplification provided by the Kalman filter.

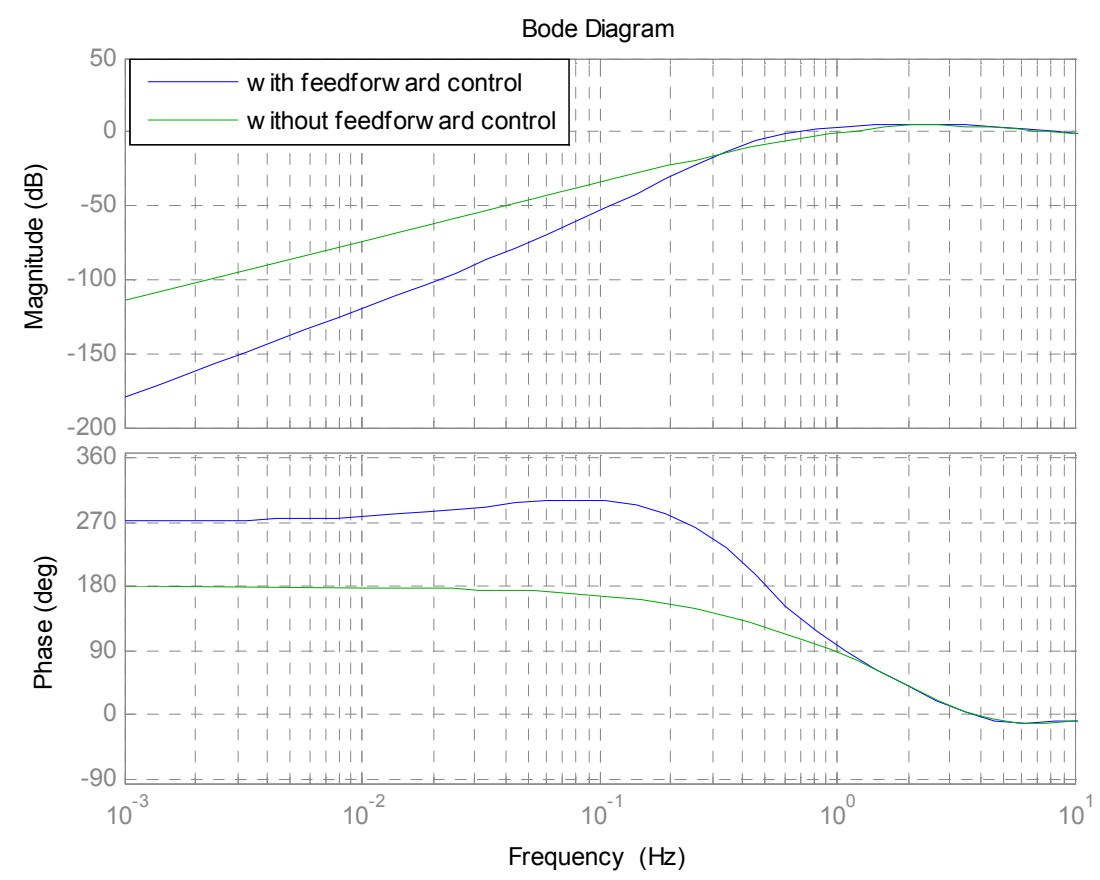

Figure 6. The sensitivity function response. 


\section{Simulations and Experiments}

Verification of this control scheme was performed through simulations and experiments.

\subsection{Simulations}

The sampling frequency of the CCD is $50 \mathrm{~Hz}$. The precision of the rate sensor is about $0.01^{\circ} / \mathrm{s}$. The target trajectory is emulated by the function $\theta=a \tan (0.22 t-6.0)$, which has the maximum velocity $12.4^{\circ} / \mathrm{s}$ and the maximum acceleration $1.9^{\circ} / \mathrm{s}$ shown in Figure 7 . This simulation only presents a single axis of the azimuth due to the similarity. The LOS error with and without feedforward control is compared in Figure 8. We can see that the maximum LOS error with the proposed method is $0.01^{\circ}$, about one-ninth of that with only feedback control.

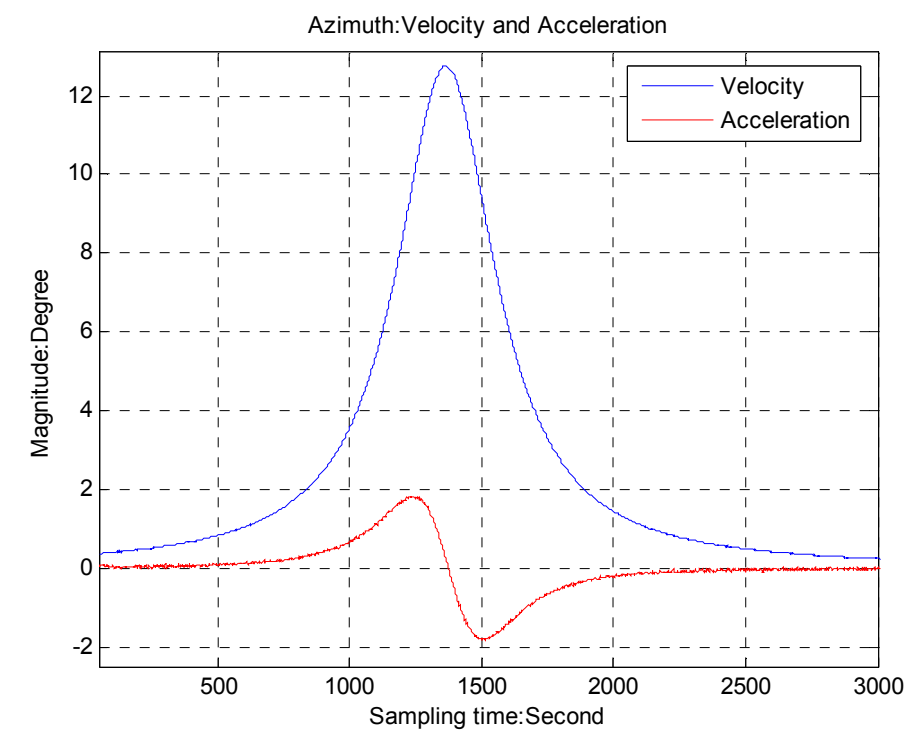

Figure 7. The simulative target velocity and acceleration.

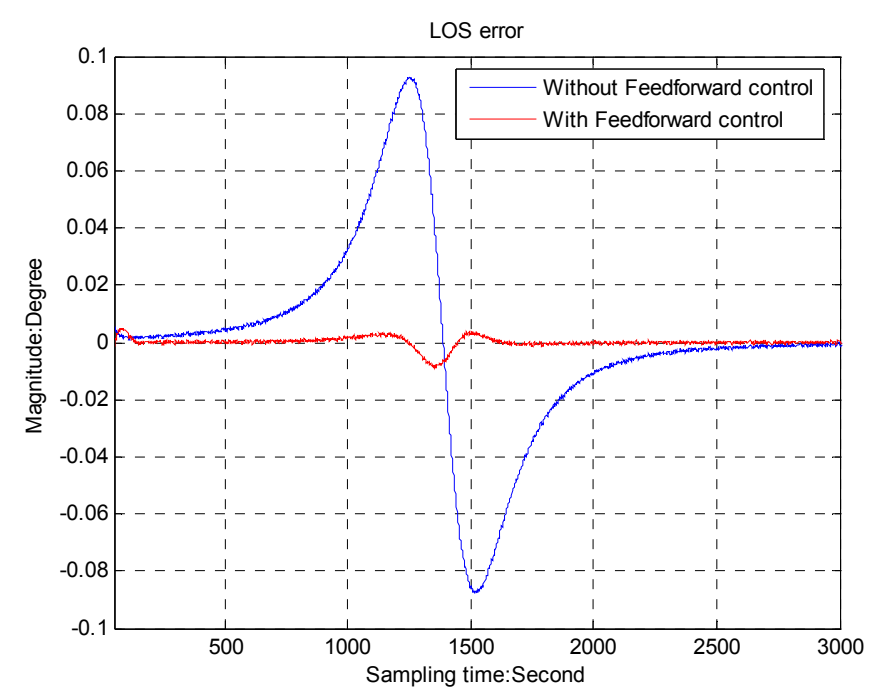

Figure 8. LOS error for the simulative target.

\subsection{Experiments}

The trajectory of a moving target is provided by dynamic moving target simulators. A two-axis gimbal system with a $50 \mathrm{~Hz}$ CCD as a tracker is used to track the moving target. The angular speed 
from the gyro is shown in Figure 9 while acceleration is differentiated by the gyro as shown in Figure 10. The period of the trajectory is about $22 \mathrm{~s}$, equivalent to $0.048 \mathrm{~Hz}$.

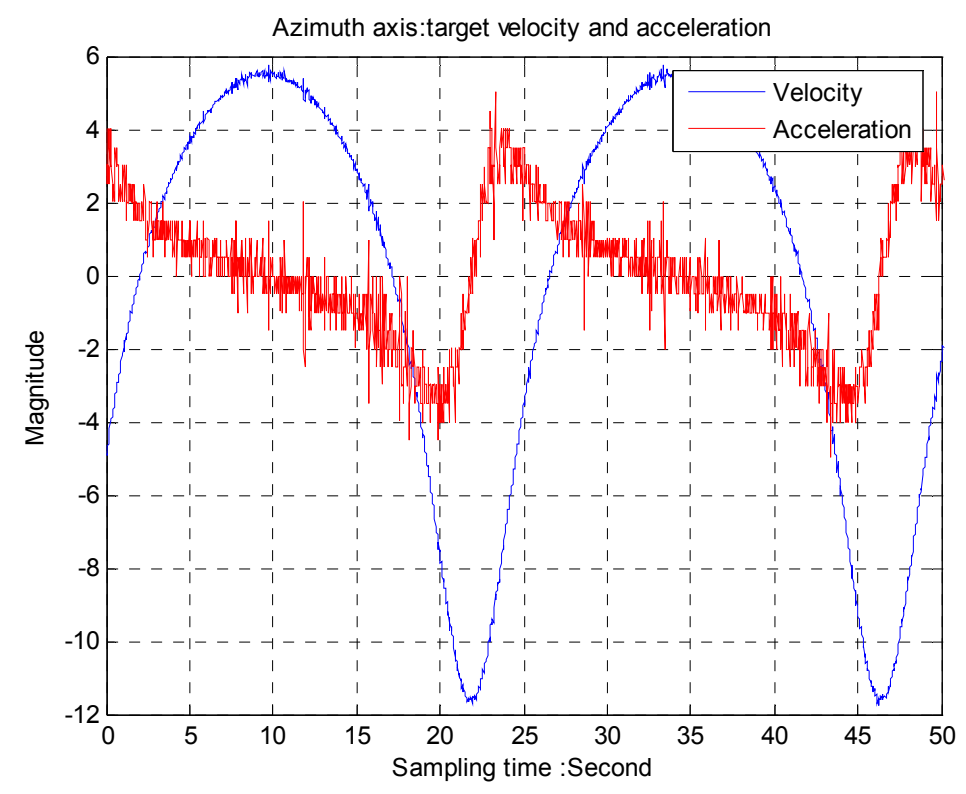

Figure 9. Azimuth axis: target velocity and acceleration.

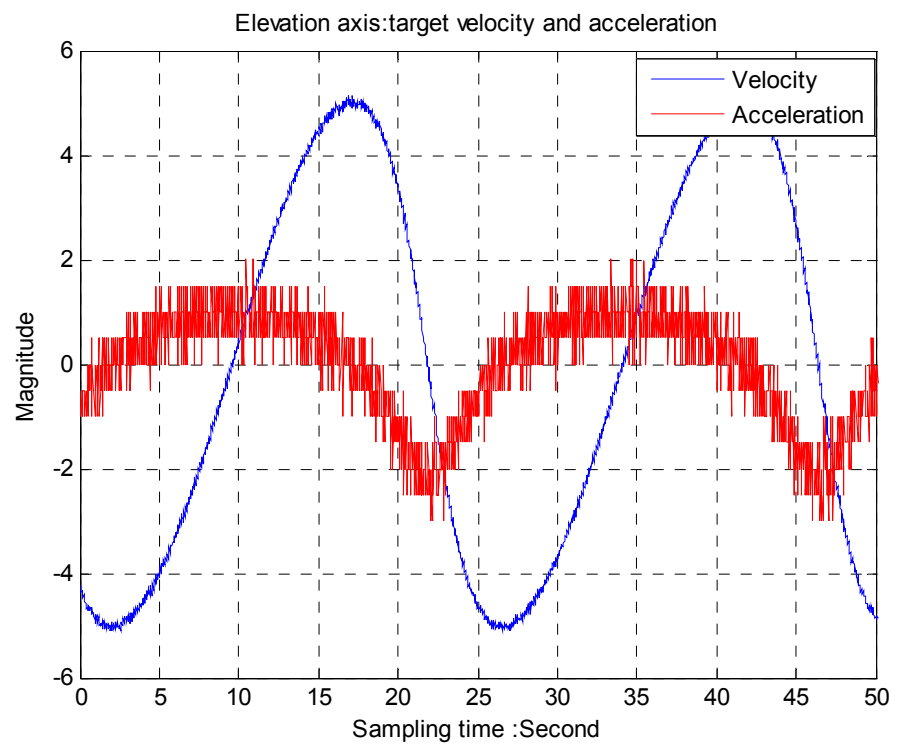

Figure 10. Elevation axis: target velocity and acceleration.

The LOS errors with the feed forward control, shown in Figure 11, are about $0.02^{\circ}$ for the azimuth axis and $0.01^{\circ}$ for the elevation axis, while we can see in Figure 12 that the LOS errors are about $0.2^{\circ}$ for the azimuth axis and $0.12^{\circ}$ for the elevation axis, respectively, without the feed forward control.

Friction deteriorates tracking performance [17], resulting in a "not smooth error", especially if the gimbal speed is low or approximates zero. It is thus important to compensate friction for a precision control system. 


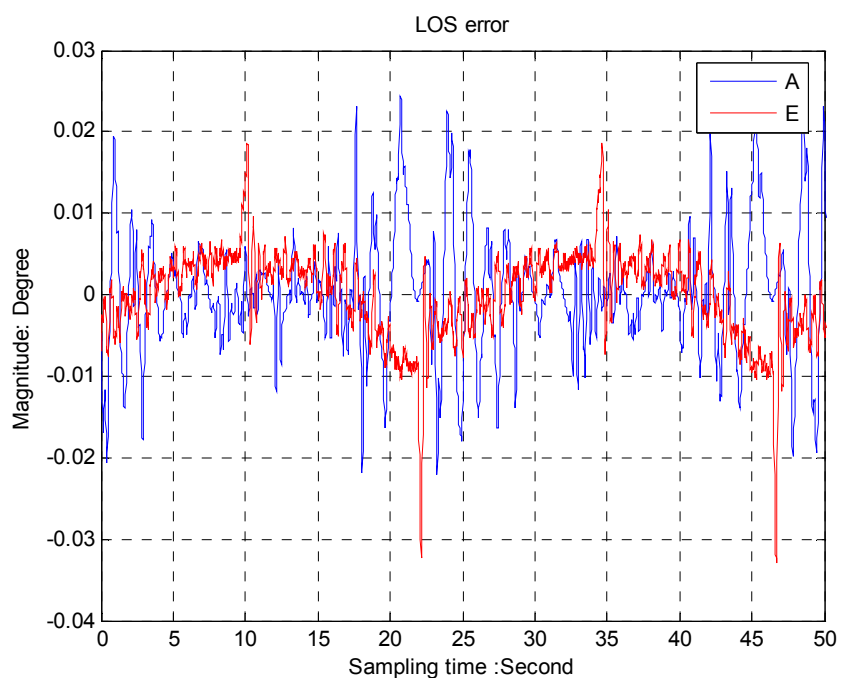

Figure 11. The LOS error with feed forward control.

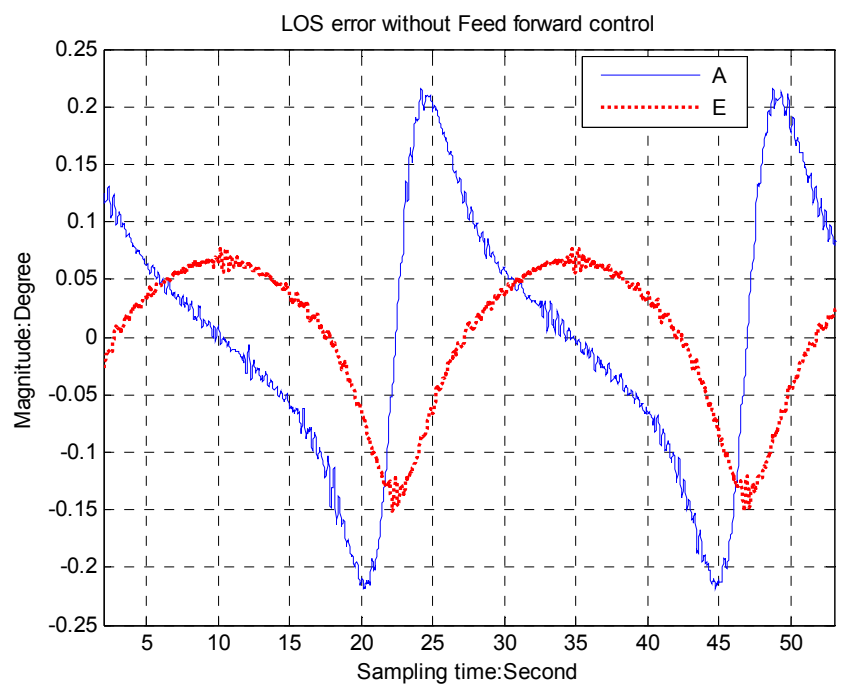

Figure 12. The LOS error without feed forward control.

\section{Conclusions}

A feed forward control based on sensor fusion for a CCD-based tracking loop is proposed to reduce some error-related time delays. In this paper, we focus on the implementation of the feed forward control, the optimization of the control parameters and the analysis of the closed-loop stability. Experiments verify the technique proposed here effectively enhances the closed-loop performance in comparison with the classical control mode. There are some other topics not covered in this paper. The first one is how to optimize the control parameters in an all-round manner rather than in an independent way; second, it is very challenging to investigate acceleration feed forward [18] to improve performance; last but not least, some experiments are required to verify this method for an inertial stabilization platform [19].

Acknowledgments: We would like to gratefully acknowledge Yu for technical support.

Author Contributions: Tao Tang is the head of the research group that conducted this study. He contributed to the research through his general guidance and advice. Daijun Zhong and Jing Tian designed the experiments; Chengyu Fu gave some advices about the experiments.

Conflicts of Interest: The authors declare no conflict of interest. 


\section{References}

1. Hutchinson, S.; Hager, G.D.; Corke, P.I. A Tutorial on Visual Servo Control. IEEE Trans. Rob. Autom. 1996, 12, 651-670. [CrossRef]

2. Dhou, S.; Motai, Y. Scale-invariant optical flow in tracking using a pan-tilt-zoom camera. Rob. Available CJO 2014. [CrossRef]

3. Motai, Y.; Jha, S.K.; Kruse, D. Human tracking from a mobile agent: Optical flow and Kalman filter arbitration. Signal Proc. Image Commun. 2012, 27, 83-95. [CrossRef]

4. Hurak, Z.; Rezac, M. Delay compensation in a dual-rate cascade visual servomechanism. In Proceedings of the 49th IEEE Conference on Decision and Control, Atlanta, GA, USA, 15-17 December 2010.

5. Fitts, J.M. Aided tracking as Applied to High Accuracy Pointing Systems. IEEE Trans. Aerosp. Electron. Syst. 1973, AES-9, 350-368. [CrossRef]

6. Downey, G.A., Jr.; Fountain, H.W.; Riding, T.J.; Eggleston, J.; Hopkins, M.; Adams, B. Sled tracking system. Proc. SPIE Acquis. Track. Pointing 1991, 40. [CrossRef]

7. Hilkert, J.M. Kinematic Algorithms for Line-of-Sight Pointing and Scanning using INS/GPS Position and Velocity Information. In Proceedings of the SPIE on Acquisition, Tracking, and Pointing XIX, Bellingham, WA, USA, 28 March 2005; Volume 5810.

8. Corke, P.I. Visual Control of Robots: High-Performance Visual Servoing. In Mechatronics; Research Studies Press: Hertfordshire, UK, 1996.

9. Ekstrand, B. Tracking Filters and Models for Seeker Applications. IEEE Trans. Aerosp. Electron. Syst. 2001, 37, 965-977. [CrossRef]

10. Waldmann, J. Line-of-Sight Rate Estimation and Linearizing Control of an Imaging Seeker in a Tactical Missile Guided by Proportional Navigation. IEEE Trans. Control Syst. Technol. 2002, 10, 556-567. [CrossRef]

11. Palumbo, N.F.; Blauwkamp, R.A.; Lloyd, J.M. Basic Principles of Homing Guidance. Johns Hopkin Apl Tech. Dig. 2010, 29, 25-41.

12. L, X.R.; Jilkov, V.P. Survey of Maneuvering Target Tracking: Dynamic Models. In Proceedings of the SPIE Conference on Signal and Data Processingof Small Targets, Orlando, FL, USA, 24 April 2000.

13. Li, X.R.; Jilkov, V.P. Survey of maneuvering target tracking. Part I. Dynamic models. In IEEE Transactions on Aerospace and Electronic Systems; IEEE: New York, NY, USA, 2003; Volume 39, pp. 1333-1364.

14. Li, X.R.; Jilkov, V.P. Survey of Maneuvering Target Tracking. Part II: Motion Models of Ballistic and Space Targets. In IEEE Transactions on Aerospace and Electronic Systems; IEEE: New York, NY, USA, 2010; Volume 46, pp. 96-119.

15. Lee, B.; Park, Y.J.B. An intelligent tracking method for a maneuvering target. Int. J. Control Autom. Syst. 2003, 1, 344-350.

16. Horowitz, R.; Li, Y.; Oldham, K.; Kon, S.; Huang, X.H. Dual-stage servo systems and vibration compensation in computer hard disk drives. Control Eng. Pract. 2007, 15, 291-305. [CrossRef]

17. Berger, E.J. Friction modeling for dynamic system simulation. Appl. Mech. Rev. 2002, 55, 535-577. [CrossRef]

18. Boerlage, M.; Steinbuch, M.; Lambrechts, P.; van de Wal, M. Model-based feedforward for motion systems. In Proceedings of 2003 IEEE Conference on Control Applications, Istanbul, Turkey, 23-25 June 2003; pp. 1158-1163.

19. Hilkert, J.M. Alpha-Theta Technol, Richardson Inertially stabilized platform technology Concepts and principles. IEEE Control Syst. Mag. 2008, 28, 26-46. [CrossRef]

(C) 2016 by the authors; licensee MDPI, Basel, Switzerland. This article is an open access article distributed under the terms and conditions of the Creative Commons Attribution (CC-BY) license (http:/ / creativecommons.org/licenses/by/4.0/). 